\title{
A question of time: tissue adaptation to mechanical forces
}

\author{
Tom Wyatt ${ }^{1,2,3,4}$, Buzz Baum ${ }^{1,4}$ and Guillaume Charras $2,4,5$ \\ 1. LMCB, 2. LCN, 3. CoMPLEX, 4. IPLS, 5. CDB, \\ UCL, Gower Street, London WC1E 6BT, UK
}

\begin{abstract}
$\underline{\text { Abstract }}$
While much attention has been focused on the force-generating mechanisms responsible for shaping developing embryos, less is known about the ways in which cells in animal tissues respond to mechanical stimuli. Forces will arise within a tissue as the result of processes such as local cell death, growth and division, but they can also be an indirect consequence of morphogenetic movements in neighbouring tissues or be imposed from the outside, for example, by gravity. If not dealt with, the accumulation of stress and the resulting tissue deformation can pose a threat to tissue integrity and structure. Here we follow the time-course of events by which cells and tissues return to their preferred state following a mechanical perturbation. In doing so, we discuss the spectrum of biological and physical mechanisms known to underlie mechanical homeostasis in animal tissues.
\end{abstract}

\section{The precarious affair of morphogenesis}

Cells live a manifold existence. They are both complex biochemical information processing entities and mechanical objects that live within mechanically active environments. As our knowledge of the molecular mechanisms underlying mechanosensation and mechanotransduction improves, these mechanical and biochemical realities appear increasingly intertwined.

The crosstalk between mechanics and biochemistry is responsible for some of the most remarkable characteristics of cellular materials [1]. This is perhaps most apparent during developmental morphogenesis when forces generated by cells working in concert re-shape tissues to give rise to complex three-dimensional architecture. The accumulation of stress within a morphogenetically active tissue, however, can carry significant risks. These include tissue fracture [2, 3], loss of cellular integrity [4] and mitotic errors [5]. Perturbation experiments clearly demonstrate the threat posed to epithelia during development. For example, reduction of the strength of cell-cell adhesion can lead to mechanical failure and the consequences of this are clear from the colourful names given to many of the adherens junction components identified by their phenotype in the fly, such as shotgun, bazooka, stardust, scribble and crumbs [6]. Similar disruptions can also be observed in vertebrates [7]. Importantly, it is the tissues engaged in 
morphogenesis that tend to fail because they are exposed to forces extrinsic and intrinsic to the tissue [8]. Since epithelial sheets often act as barriers to separate the organism from the outside world, these types of failure can have devastating effects.

To guard against such risks, tissues have evolved a wide variety mechanisms that help to limit the occurrence and consequences of mechanical failure. For example, exposure of embryonic tissues to high stresses can lead to cell fusion, a process that has been proposed to prevent tissue fracture [4, 9]. Mechanical perturbations of tissues, however, vary vastly in time-scale - from the sub-second twitches of muscles to slow embryonic growth, which can proceed over many months. Here, we will discuss mechanisms that act over this broad range of timescales to maintain tissue homeostasis, exploring how cellularised tissues respond to extrinsic force and deformation to guide their return to the preferred mechanical state.

\section{The material properties of living matter and the impact of rapid physical perturbations}

We first examine the fastest interactions and the most immediate responses and then later examine progressively longer time-scales. An extreme example of a rapid mechanical perturbation to a biological system is the strike of the Mantis shrimp's "club". Moving at $\sim 23 \mathrm{~m} / \mathrm{s}$, this hammer-like structure fractures the shells of the clams and crabs on which the shrimp preys with forces of $\sim 500 \mathrm{~N}$, applied over a fraction of a millisecond [10]. At such short time-scales, mechanochemical signalling and genetic regulation are of no consequence. How then does the predator protect itself from the impact of these strikes? The answer lies in the material properties of the club itself [11]. Accordingly, the animal has evolved a complex composite mineral armature which shields the club against crack propagation [10-12].

Similarly, the immediate impact of sudden mechanical perturbations on any soft animal tissues will be governed by their material properties. To a large extent, this will be dictated by molecular assemblies such as the cytoskeleton and lipid membranes. As a result, alterations in the mechanical properties and organisation of these structures can lead to disease. Indeed, loss of a single structural protein, Dystrophin, can mechanically weaken muscle cells and is a likely cause of stress-dependent muscle degeneration in many cases of muscular dystrophy [13].

Even at short time-scales (milliseconds to seconds), cellularised materials display highly complex material properties which are still not fully understood. The rapid redistribution of cytosol through networks of organelles and the cytoskeleton at sub-second time-scales is thought to give rise to poro-elastic behaviour (Fig. 1A) [14]. Then, over the course of seconds, scale-free power-law rheology [15, 16], strain stiffening [17], and fluidisation [17, 18] are observed [19]. 
The complexity of these mechanical behaviours is hardly surprising given the rich mechanical behaviour that can be observed in studies of even single isolated cytoskeletal filaments exposed to mechanical perturbation [20, 21]. For instance, the cyclic bending of a single microtubule gradually reduces its stiffness by inducing flaws in the crystal lattice of tubulin heterodimers [22]. For filamentous networks, the observed physical behaviours are, of course, even richer [23] and their rheology strongly influences the mechanical behaviour of cells and tissues. For example, Etienne et al. [24] recently showed that collective dynamics of actin filaments and myosins enables the actomyosin cortex to instantaneously sense and adapt to external changes in substrate stiffness.

Another immediate effect of a sudden mechanical perturbation is deformation of the plasma membrane and the endomembrane system of the tissue's cells. The plasma membrane has a large elastic modulus but can only withstand $2-3 \%$ stretch before lysis [25], so stretching causes high stress and risks rupture. To avoid this, reserves of membrane are stored in folds that flatten upon stretch [26]. Conversely, when surface area is suddenly reduced, excess plasma membrane forms tubules whose initial shape can be accurately predicted from physical considerations [27].

\section{Turnover of protein assemblies}

At minute time-scales the turnover of proteinaceous structures that bear the load of externally applied stress will function as a ubiquitous source of stress dissipation (Fig. 1B) [28]. This is especially the case for the large-scale cytoskeletal networks of filaments that connect cells to their environment. Here, protein turnover will cause the network as a whole to adapt to the new mechanical environment through the remodelling of stress-bearing structures [29]. This process can be actively enhanced by the action of associated motor proteins [29, 30]. Although these fundamental features of animal cell biology have diverse molecular origins, they surely act as a first line of defence against the dangers of acute or prolonged stress by ensuring that internal stresses are rapidly redistributed and relaxed.

\section{Intermediate time-scale responses stem from cellular processes}

Over longer periods of time, of minutes to hours, many of the behaviours that we usually associate with living systems first become apparent. This is the timeframe over which active cellular behaviours begin to play a role in stress dissipation and in dealing with the consequences of changes in cell structure that arise from an imposed deformation. So, for example, it is over the course of minutes that the flaws in the crystal lattice of a microtubule subjected to cycles of bending can self-heal through the incorporation of new tubulin heterodimers [22]. Accompanying the molecular events that occur at this time-scale are a wide 
variety of cellular behaviours, all of which can function to regulate tissue stress. These include cell neighbour exchange, cell division, cell fusion and cell extrusion.

By exchanging neighbours, cells are able to translocate within an epithelium (Fig. $1 \mathrm{C})$. Depending on the boundary conditions of the tissue, these rearrangements can alter cell and tissue shape as well as local patterns of stress. The process relies on the ability of cells to add or remove specific cell-cell interfaces via the regulation of junction tension and adhesion [31-33]. In the best-studied examples, a sophisticated developmental program polarises neighbour exchange events to reshape the developing fly embryo [31, 33, 34]. However, neighbour exchange can also be stimulated by forces external to a tissue, such as those induced in the Drosophila wing disc by the constriction of the neighbouring wing hinge [35]. Recent studies suggest that neighbour exchanges induced by external forces applied by neighbouring tissues may be a generic behaviour during development $[36,37]$.

Cell division can have an effect analogous to neighbour exchange on tissue stress (Fig. 1D) [4, 38]. It is sometimes assumed that division simply cuts the mother cell mass in two. If this were the case, division would not necessarily dissipate stress [4]. Rather, recent work has shown that the shape changes taking place during mitotic rounding and anaphase elongation enable division to redistribute the mother cell's mass, causing a dilation along the axis of division and a contraction in the perpendicular direction [38]. This makes the orientation of division critical. In individual cells, it has long been known that divisions are oriented along the long axis of interphase cell shape [39-41]. While the mechanisms involved are not fully understood, extrinsically applied stress has also been implicated [42]. In tissues, the application of an anisotropic strain induces alignment of the cell long axes in the principal strain direction, leading to oriented divisions that facilitate the flow of cellular material to dissipate stress, eventually restoring isotropic cell shape [38]. The fact that divisions appear to orient in response to stretch in many developmental systems suggests that this may represent a generic mechanism [43-45].

The view that cell divisions form a central part of the mechanism underpinning epithelial homeostasis is further supported by the well-established roles of mechanical stress in altering the rate of cell cycle progression [46]. More recently, Streichan et al. [47] re-examined the role of the G1-S-phase checkpoint that is sensitive to spatial constraints, which triggers cell cycle progression if space becomes available or if the tissue is stretched. In similar experiments, the initiation of DNA replication in response to stretch has been shown to require the nuclear translocation of Yap1 and $\beta$-catenin, together with intact extracellular Ecadherin adhesion, implying the action of mechanisms to directly sense and react to stress or the resulting changes in cell shape [48].

Tissues can also respond to localized decreases in tension. For example, as a 
consequence of a developmentally regulated process of tissue fusion, cells in the midline of the developing fly notum experience local overcrowding. This leads to cell extrusion (Fig. 1E), which over time restores normal cell packing and resting tissue tension [49]. Similar effects have also been observed in mammalian epithelia in response to a sudden increase in cell density [50].

Although the cell behaviours discussed here differ from one-another, they make complementary contributions to mechanical homeostasis. For example, both division and neighbour exchange are oriented processes, which alter cell shape and tissue stress in comparable manners, allowing for the redistribution of cellular material in response to an applied stress. Moreover, these cell-level behaviours can compensate for one another. Thus, a failure of intercalation in fly embryos is compensated by more extreme changes in cell shape [37], while the misorientation of divisions during zebrafish epiboly leads to cell-cell fusion [4]. Cell fusion may represent a temporary short-term solution to avoid tissue rupture (as seen following wounding in fly larvae [9]) but is puzzling as it can only represent a short-term solution since it necessitates multinucleate cell clearance at a later stage. Together, these cellular-scale processes can combine to give rise to tissue flow [35, 51-53]. In the future, it will be important to understand why one process is preferred over another in particular instances. However, the observation that the final structure of the fly wing varies less than the relative contributions of oriented division and neighbour exchange suggests that the mechanisms are to some extent interchangeable [35].

\section{Adaptation at long time-scales}

The longest time-scales, of days to years, present a new set of challenges for developing organisms. At the furthest end of the spectrum, an organism must generate and retain its complex 3D form in the presence of gravity, whose mechanical influence is felt through an entire lifetime. In soft vertebrate tissues this feat is achieved, in part, via the establishment of an appropriate level of constitutive actomyosin tissue tension under the control of YAP [54] - in the absence of which animal bodies were found to sag and tissues misalign (Fig. 1F).

Since cellular tissues behave like viscous fluids over long time-scales, a constitutive tension might be expected to drive flows to achieve a reduction in surface area - transforming tissue structures into featureless balls. This can be prevented, for example, by balancing constitutive tension with a preferred cell shape, or by attaching tissues to less viscous structures, such as the extracellular matrix or bone. In general, the combination of stable boundaries and patterned tension generation can robustly guide tissue morphogenesis, as in the Drosophila wing disc where patterned anchoring of the epithelium to the cuticle serves such a purpose $[35,55]$. 
Mechanosensation and Mechanotransduction: what are the relevant timescales?

The above discussion contains an omission. An important class of protective adaptations to mechanical perturbations are responses that depend on mechanically-initiated biomolecular signalling. This is a very active area of research, which was pioneered by workers interested in hearing [56]. Mechanochemical triggers include the stress-induced opening of an ion-channel [57] or the unfolding of stress-bearing proteins in the system [58, 59]. Such mechanotransduction can stimulate the local reinforcement of focal adhesions [58, 60-62] and adherens junctions [59, 63-66], which could function to protect tissues from high levels of stress. As discussed above, however, in living cells molecular turnover within force-bearing cellular substructures is almost ubiquitous and will rapidly relax most stresses [3]. Thus, channel opening and protein unfolding can only monitor stresses that arise at shorter time-scales than molecular turnover, limiting their importance in many morphogenetic processes (which act over periods of minutes to days) unless they are functioning as components of a homeostatic system.

For this reason it remains unclear, for example, how a constant imposed stress can trigger proliferation in surrounding cells via $\beta$-catenin signalling over the course of weeks and months [67]. One possibility is that stress is stored over long time-scales in a less viscous structure than the cells, such as the extracellular matrix. Cell shape deformation could also serve as a memory of an applied force, lasting long after turnover has dissipated the initial stress. However, as explained above, over a period of hours to days the processes of cell growth, division, neighbour exchange and extrusion will tend to homeostatically restore cell shape as well as remove tissue stresses.

\section{Conclusion}

In cellularised tissues, robust responses to a mechanical perturbation require the action of appropriate homeostatic mechanisms at multiple time-scales. The material properties of tissues must ensure resistance to fracture at short timescales and cellular processes such as division, neighbour exchange and extrusion can remodel tissue shape over minutes and hours. The majority of any imposed stress is likely to be rapidly dissipated by cytoskeleton turnover and membrane dynamics, even if some stress may remain at long time scales $[3,38]$, especially when stored in an extracellular material. This residual stress, along with deformations of cell shape, may act to guide the restoration of tissue homeostasis. Importantly, as we have argued here, although there is currently great interest in the molecular machinery that translates force into a biochemical signal at adhesion sites, this is likely to make only a small contribution to the entire spectrum of responses that maintain mechanical tissue homeostasis. Thus, understanding morphogenesis is challenging because it requires understanding 
many processes with time- and length-scales spanning many orders of magnitude (Fig. 1G). Distinguishing between the complex emergent physical properties of living matter and the equally complex mechanoresponsive behaviours of cells is crucial and presents an exciting challenge for the present and future.

\section{Acknowledgements}

TW is part of the EPSRC funded doctoral training program (CoMPLEX). BB thanks UCL and Cancer Research UK and the BBSRC. GC is supported by the BBSRC (BB/M003280) and TW is supported by a consolidator grant from the European Research Council to GC (MolCellTissMech, 647186). We would like to thank Nargess Khalilgharibi, Mark Miodownik, Yanlan Mao and Ys for useful discussions and feedback.

\section{References}

1. Vincent, J., Structural Biomaterials. 2012: Princeton University Press.

2. Casares, L., et al., Hydraulic fracture during epithelial stretching. Nat Mater, 2015. 14(3): p. 343--351.

3. Harris, A.R., et al., Characterizing the mechanics of cultured cell monolayers. Proceedings of the National Academy of Sciences, 2012.

4. Campinho, P., et al., Tension-oriented cell divisions limit anisotropic tissue tension in epithelial spreading during zebrafish epiboly. Nat Cell Biol, 2013. 15(12): p. 1405--1414.

5. $\quad$ Lancaster, O.M., et al., Mitotic Rounding Alters Cell Geometry to Ensure Efficient Bipolar Spindle Formation. Developmental Cell, 2013. 25(3): p. 270 283.

6. Müller, H.A. and E. Wieschaus, armadillo, bazooka, and stardust are critical for early stages in formation of the zonula adherens and maintenance of the polarized blastoderm epithelium in Drosophila. The Journal of Cell Biology, 1996. 134(1): p. 149-163.

7. Levine, E., et al., Selective disruption of E-cadherin function in early Xenopus embryos by a dominant negative mutant. Development, 1994. 120(4): p. 901-909.

8. Tepass, U., et al., shotgun encodes Drosophila E-cadherin and is preferentially required during cell rearrangement in the neurectoderm and other morphogenetically active epithelia. Genes Dev, 1996. 10(6): p. 672-85.

9. Losick, V.P., D.T. Fox, and A.C. Spradling, Polyploidization and cell fusion contribute to wound healing in the adult Drosophila epithelium. Current Biology, 2013. 23(22): p. 2224--2232.

10. Patek, S.N. and R.L. Caldwell, Extreme impact and cavitation forces of a biological hammer: strike forces of the peacock mantis shrimp Odontodactylus scyllarus. Journal of Experimental Biology, 2005. 208(19): p. 3655--3664. 
11. Amini, S., et al., The role of quasi-plasticity in the extreme contact damage tolerance of the stomatopod dactyl club. Nat Mater, 2015. 14(9): p. 943--950.

12. Weaver, J.C., et al., The Stomatopod Dactyl Club: A Formidable DamageTolerant Biological Hammer. Science, 2012. 336(6086): p. 1275-1280.

13. Rahimov, F. and L.M. Kunkel, Cellular and molecular mechanisms underlying muscular dystrophy. The Journal of Cell Biology, 2013. 201(4): p. 499-510.

14. Moeendarbary, E., et al., The cytoplasm of living cells behaves as a poroelastic material. Nat Mater, 2013. 12(3): p. 253--261.

15. Laudadio, R.E., et al., Rat airway smooth muscle cell during actin modulation: rheology and glassy dynamics. Am J Physiol Cell Physiol, 2005. 289(6): p. C1388-95.

16. Hildebrandt, J., Comparison of mathematical models for cat lung and viscoelastic balloon derived by Laplace transform methods from pressurevolume data. Bull Math Biophys, 1969. 31(4): p. 651-67.

17. Trepat, X., et al., Universal physical responses to stretch in the living cell. Nature, 2007. 447(7144): p. 592-5.

18. Kollmannsberger, P., C.T. Mierke, and B. Fabry, Nonlinear viscoelasticity of adherent cells is controlled by cytoskeletal tension. Soft Matter, 2011. 7(7): p. 3127-3132.

19. Kollmannsberger, P. and B. Fabry, Linear and Nonlinear Rheology of Living Cells. Annual Review of Materials Research, 2011. 41(1): p. 75-97.

20. Galkin, V.E., A. Orlova, and E.H. Egelman, Actin filaments as tension sensors. Curr Biol, 2012. 22(3): p. R96-101.

21. Romet-Lemonne, G. and A. Jegou, Mechanotransduction down to individual actin filaments. Eur J Cell Biol, 2013. 92(10-11): p. 333-8.

22. Schaedel, L., et al., Microtubules self-repair in response to mechanical stress. Nat Mater, 2015. 14(11): p. 1156-63.

23. Schaller, V., et al., Polar patterns of driven filaments. Nature, 2010. 467(7311): p. 73-7.

24. Etienne, J., et al., Cells as liquid motors: mechanosensitivity emerges from collective dynamics of actomyosin cortex. Proc Natl Acad Sci U S A, 2015. 112(9): p. 2740-5.

25. Hochmuth, R.M., N. Mohandas, and P.L. Blackshear, Jr., Measurement of the elastic modulus for red cell membrane using a fluid mechanical technique. Biophys J, 1973. 13(8): p. 747-62.

26. Gauthier, N.C., et al., Temporary increase in plasma membrane tension coordinates the activation of exocytosis and contraction during cell spreading. Proc Natl Acad Sci U S A, 2011. 108(35): p. 14467-72.

27. Kosmalska, A.J., et al., Physical principles of membrane remodelling during cell mechanoadaptation. Nat Commun, 2015. 6.

28. Sedzinski, J., et al., Polar actomyosin contractility destabilizes the position of the cytokinetic furrow. Nature, 2011. 476(7361): p. 462-6.

29. Mizuno, D., et al., Nonequilibrium Mechanics of Active Cytoskeletal Networks. Science, 2007. 315(5810): p. 370-373.

30. Wilson, C.A., et al., Myosin II contributes to cell-scale actin network treadmilling through network disassembly. Nature, 2010. 465(7296): p. 373-7. 
31. Simoes, S.d.M., A. Mainieri, and J.A. Zallen, Rho GTPase and Shroom direct planar polarized actomyosin contractility during convergent extension. The Journal of Cell Biology, 2014. 204(4): p. 575-589.

32. Warrington, S.J., H. Strutt, and D. Strutt, The Frizzled-dependent planar polarity pathway locally promotes E-cadherin turnover via recruitment of RhoGEF2. Development, 2013. 140 (5): p. 1045--1054.

33. Bardet, P.-L., et al., PTEN Controls Junction Lengthening and Stability during Cell Rearrangement in Epithelial Tissue. Developmental Cell, 2013. 25(5): p. 534 - 546.

34. Kasza, K.E., D.L. Farrell, and J.A. Zallen, Spatiotemporal control of epithelial remodeling by regulated myosin phosphorylation. Proceedings of the National Academy of Sciences, 2014. 111(32): p. 11732-11737.

35. Etournay, R., et al., Interplay of cell dynamics and epithelial tension during morphogenesis of the Drosophila pupal wing. eLife, 2015. 4.

36. Collinet, C., et al., Local and tissue-scale forces drive oriented junction growth during tissue extension. Nat Cell Biol, 2015. 17(10): p. 1247--1258.

37. Lye, C.M., et al., Mechanical Coupling between Endoderm Invagination and Axis Extension in Drosophila. PLoS Biol, 2015. 13(11): p. e1002292.

38. Wyatt, T.P.J., et al., Emergence of homeostatic epithelial packing and stress dissipation through divisions oriented along the long cell axis. Proceedings of the National Academy of Sciences, 2015. 112(18): p. 5726-5731.

39. Gibson, W.T., et al., Control of the Mitotic Cleavage Plane by Local Epithelial Topology. Cell, 2011. 144(3): p. 427--438.

40. Hertwig, O., About the value of the first cleavage for the organisation of the embryo. Experimental studies on frog and triton eggs. Arch. Mikr. Anat, 1893. xlii: p. $662--807$.

41. Minc, N., D. Burgess, and F. Chang, Influence of Cell Geometry on Division-Plane Positioning. Cell, 2011. 144(3): p. 414 - 426.

42. Fink, J., et al., External forces control mitotic spindle positioning. Nat Cell Biol, 2011. 13(7): p. 771--778.

43. da Silva, S.M. and J.-P. Vincent, Oriented cell divisions in the extending germband of Drosophila. Development, 2007. 134(17): p. 3049--3054.

44. Mao, Y., et al., Differential proliferation rates generate patterns of mechanical tension that orient tissue growth. The EMBO Journal, 2013. 32(21): p. 2790-2803.

45. LeGoff, L., H. Rouault, and T. Lecuit, A global pattern of mechanical stress polarizes cell divisions and cell shape in the growing Drosophila wing disc. Development, 2013. 140(19): p. 4051-4059.

46. Davies, P.F., et al., Turbulent fluid shear stress induces vascular endothelial cell turnover in vitro. Proceedings of the National Academy of Sciences, 1986. 83(7): p. 2114-2117.

47. Streichan, S.J., et al., Spatial constraints control cell proliferation in tissues. Proceedings of the National Academy of Sciences, 2014. 111(15): p. 55865591. 
48. Benham-Pyle, B.W., B.L. Pruitt, and W.J. Nelson, Mechanical strain induces Ecadherin--dependent Yap1 and $\beta$-catenin activation to drive cell cycle entry. Science, 2015. 348(6238): p. 1024-1027.

49. Marinari, E., et al., Live-cell delamination counterbalances epithelial growth to limit tissue overcrowding. Nature, 2012. 484(7395): p. 542--545.

50. Eisenhoffer, G.T., et al., Crowding induces live cell extrusion to maintain homeostatic cell numbers in epithelia. Nature, 2012. 484(7395): p. 546--549.

51. Tlili, S., et al., Colloquium: Mechanical formalisms for tissue dynamics. The European Physical Journal E, 2015. 38(5): p. 1--31.

52. Delarue, M., et al., Mechanical Control of Cell flow in Multicellular Spheroids. Physical Review Letters, 2013. 110(13): p. 138103.

53. Ranft, J., et al., Fluidization of tissues by cell division and apoptosis. Proceedings of the National Academy of Sciences, 2010. 107(49): p. 2086320868.

54. Porazinski, S., et al., YAP is essential for tissue tension to ensure vertebrate $3 D$ body shape. Nature, 2015. 521(7551): p. 217--221.

55. Ray, R.P., et al., Patterned Anchorage to the Apical Extracellular Matrix Defines Tissue Shape in the Developing Appendages of Drosophila. Developmental Cell, 2015. 34(3): p. 310 - 322.

56. Békésy, G., Concerning the pleasures of observing, and the mechanics of the inner ear. Les Prix Nobel en, 1961: p. 184-208.

57. Sukharev, S. and F. Sachs, Molecular force transduction by ion channels: diversity and unifying principles. J Cell Sci, 2012. 125(Pt 13): p. 3075-83.

58. Yao, M., et al., Mechanical activation of vinculin binding to talin locks talin in an unfolded conformation. Sci Rep, 2014. 4: p. 4610.

59. Yao, M., et al., Force-dependent conformational switch of $\alpha$-catenin controls vinculin binding. Nat Commun, 2014. 5.

60. Fouchard, J., et al., Three-dimensional cell body shape dictates the onset of traction force generation and growth of focal adhesions. Proc Natl Acad Sci U S A, 2014. 111(36): p. 13075-80.

61. Lee, S.E., R.D. Kamm, and M.R. Mofrad, Force-induced activation of talin and its possible role in focal adhesion mechanotransduction. J Biomech, 2007. 40(9): p. 2096-106.

62. Sawada, Y., et al., Force sensing by mechanical extension of the Src family kinase substrate p130Cas. Cell, 2006. 127(5): p. 1015-26.

63. Leerberg, J.M., et al., Tension-Sensitive Actin Assembly Supports Contractility at the Epithelial Zonula Adherens. Current Biology, 2014. 24(15): p. 1689 - 1699.

64. Buckley, C.D., et al., The minimal cadherin-catenin complex binds to actin filaments under force. Science, 2014. 346(6209).

65. Borghi, N., et al., E-cadherin is under constitutive actomyosin-generated tension that is increased at cell--cell contacts upon externally applied stretch. Proceedings of the National Academy of Sciences, 2012. 109(31): p. 1256812573.

66. Yonemura, S., et al., a-Catenin as a tension transducer that induces adherens junction development. Nat Cell Biol, 2010. 12(6): p. 533--542. 
67. Fernandez-Sanchez, M.E., et al., Mechanical induction of the tumorigenic betacatenin pathway by tumour growth pressure. Nature, 2015. 523(7558): p. 92-95.

\section{"Special interest" papers:}

(Pare and Zallen Nature 2014) In this study, the authors identify overlapping stripes of three Toll family receptors as key regulators of intercalation during Drosophila germband extension.

(Collinet and Lecuit NCB 2015) The authors demonstrate that tissue-scale tension in the Drosophila embryo participates in orienting the elongation of newly formed junctions, thereby contributing to intercalation and germband extension.

(Bardet and Bellaiche Dev. Cell 2013) This study identifies the tumour suppressor PTEN as a regulator of junctional lengthening following cell neighbour exchange in the Drosophila wind disc. Without PTEN, myosin remains at junctions causing them to contract rather than stabilise.

(Etournay and Eaton eLife 2015) The authors investigate the role of intrinsically and extrinsically generated tension in shaping the developing Drosophila wing and reveal a role for patterned extra-cellular matrix adhesions.

(Porazinski and Heisenberg Nature 2015) A medaka fish mutant reveals the role of constitutive tension in forming shape and aligning tissues against the force of gravity.

(Campinho and Heisenberg NCB 2013) The authors show that divisions oriented along the principal axis of tension during epiboly in the zebrafish embryo act to reduce tension anisotropy and prevent cell fusions.

(Wyatt PNAS 2015) The authors demonstrate that cell divisions in a stretched epithelial monolayer are oriented with the cell shape and act to redistribute the mother cell mass and restore cell shape.

(Streichan and Hufnagel PNAS 2014) The authors apply stretch to MDCK monolayers via a stretchable substrate and find a G1-S phase checkpoint regulated by spatial constraints.

\section{Figure legends}

Figure 1. Mechanisms of tissue mechanical homeostasis act over a spectrum of time-scales. (A) The poroelastic response of cells and tissues arises from cytosol redistribution through a dense meshwork of cytoplasmic 
fibres (red) and organelles (yellow). (B) Turnover and disassembly of stress bearing structures due to the unbinding of proteins leads to a relaxation of stress. (C) Cell neighbour exchange, via the removal and addition of cell-cell junctions, can alter tissue stress patterns as well as cell and tissue shape. (D) Oriented cell divisions relax stress along the orientation of division and can help restore cell shape after an anisotropic stretch. (E) Cells can be extruded from a tissue, thereby reducing cell density in response to compression or overcrowding. $(F)$ Constitutive tissue tension is important for generating and maintaining form over long time-scales and against the force of gravity. (G) Different mechanisms of mechanical homeostasis act over a large range of time-scales. 